\title{
MITOS Y REALIDADES DE LA "IGUALDAD DE OPORTUNIDADES"
}

Enriqueta Zúñiga Chaves

El propósito de este artículo es reflexionar sobre la situación que viven todos los días los alumnos que se encuentran cursando una carrera universitaria y tienen necesidades educativas especiales asociadas a una discapacidad. Especial atención queremos darle a la contribución que la Universidad debería ofrecer en ese proceso de formación en donde el alumno adquiere sus habilidades profesionales más específicas. Es durante esta etapa de su educación cuando las y los jóvenes, las y los adultos jóvenes se apropian más conscientemente de algunos conocimientos tanto como de algunos procedimientos y desarrollan valores $y$ actitudes muy importantes para el desempeño de su profesión.

Partimos de los siguientes supuestos teóricos: primero, que la educación es para todos, de que ésta es un derecho 
universal y la Universidad tiene la obligación de procurar una educación de la mejor calidad para que todos sus alumnos, tengan éstas necesidades educativas ordinarias o más específicas; segundo, que el currículo, definido como una declaración de intenciones educativas puestas en práctica, es la herramienta para la promoción del desarrollo educativo; tercero, que cada ser humano tiene un desarrollo particular de sus capacidades y éstas, cualquiera que sea, deben ser conocidas a profundidad por los docentes, especialmente cuando generan necesidades educativas especiales que requieren a su vez de una atención y apoyo pedagógico distinto del que se ofrece a la mayoría de los alumnos y alumnas. La modificación en la planificación y en el desempeño docente utilizada por el profesor cuando un alumno necesita apoyo específico, ya sea por sus capacidades o por sus dificultades en el aprendizaje, es lo que llamamos adecuación curricular.

El procedimiento del diseño, desarrollo y aplicación de la adecuación al currículo es uno de los grandes retos que se le presenta hoy en día al docente universitario.

Por esta razón, queremos presentar algunas ideas de cómo se llevan a cabo las adecuaciones al currículum universitario, que permiten atender las necesidades educativas de los alumnos que por sus capacidades o sus dificultades para aprender, se encuentran en desventaja y requieren de apoyos específicos para compensar estas desventajas y disfrutar de la oportunidad de educarse y de procurarse una mejor calidad de vida. Además, deseamos establecer la posible relación que existiría entre estas adecuaciones al currículo universitario y la posibilidad de que los futuros profesionales, interioricen en una forma natural y vivencial, la obligada necesidad de los seres humanos de entendernos y 
aceptarnos diversos, esta diversidad como un producto directo de las diferencias que presentamos todas las personas, no solamente las que presentan una discapacidad. Es importante destacar que el hecho educativo es muy complejo y multifactorial y no bastará solamente con aplicar los conocimientos técnicos. Por otra parte, la acción didáctica se caracteriza específicamente por ser un encuentro personal asimétrico, en el cual todas las dimensiones que caracterizan a los actores involucrados se ven en mayor o menor grado implicadas.

Si lográramos incluir en las aulas universitarias el análisis de nuestra diversidad humana y si en forma natural intentáramos ser coherentes con el principio fundamental, de que la educación es un derecho universal y si también en forma natural, planificáramos nuestra forma de entregar la docencia, con temáticas y actividades diversificadas sólo tomando en consideración que nuestros alumnos, sin importar el número, serán diversos porque son "normales" y serán diversos porque naturalmente diversos son los grupos de donde provienen. Solamente en un marco institucional sensible a la diversidad del alumnado, a sus necesidades educativas, encontrará la adaptación curricular el terreno fértil para su elaboración y desarrollo. Lo contrario también podría ser cierto, en un terreno poco abonado y no fértil en cuanto a la elaboración y el desarrollo de la flexibilidad curricular, tendríamos necesariamente una institución poco o nada sensible a la diversidad humana.

Estamos hablando de una diversidad humana, que puede ser identificada por diferencias de género, de edad, de raza, de religión, de condición social, de interés, de motivación, de aptitudes, de capacidades, de oportunidades, etc. Si lográramos además, en nuestras aulas universitarias conocer 
e intentar una convivencia armónica y natural con esta diversidad, es posible, entonces, que surgieran las adecuaciones o modificaciones al currículo en una forma también natural, únicamente como parte de un proceso. Parecería entonces lógico que si nuestros alumnos universitarios, futuros profesionales en todas las áreas del saber, cuando se encuentren en el desempeño de sus labores, ofreciendo algún servicio a esa sociedad, que es también diversa, puedan en forma natural, porque así fueron sus vivencias universitarias como parte de su formación profesional, atender o brindar apoyo adecuado a las características y necesidades de los miembros de esa sociedad.

¿Quiénes son los alumnos con necesidades educativas en la Universidad y cuáles son sus características?

Todos los estudiantes matriculados en la Universidad, manifiestan algún tipo de necesidad educativa, además, todos los seres humanos tenemos necesidad de educarnos en diversas áreas. Ese es al fin, el objetivo fundamental a lograr en estos y otros centros de enseñanza. Como son tantos los estudiantes y tan diversas sus necesidades de formación, igualmente los estudiantes se atienden en diversas Unidades Académicas, en diversas carreras, en diversos cursos, con diversos planes de estudio y muy diversos profesores. Entre tanta diversidad también tenemos alumnas y alumnos cuya diversidad se nos hace más visible pues ellos o ellas presentan alguna discapacidad o dificultad para aprender.

Es muy importante para brindar apoyo a una población estudiantil, conocer cuántos son realmente los y las estudiantes matriculados(as) en la Universidad, cuáles son sus características y cuáles son sus necesidades de formación, e igualmente, pero será también muy importante determinar 
cuáles son sus necesidades educativas especiales y si están asociadas a una discapacidad. En general, conviene saber qué tipo de necesidades presentan y qué tipo de apoyo requieren; además, debemos saber en qué carreras o unidades académicas se encuentran ellos y ellas matriculados (as). Es muy importante recordar que las necesidades educativas forman parte de un continuo de necesidades educativas, que se extienden desde niveles ordinarios hasta necesidades muy significativas y que ubicadas en el tiempo, estas necesidades podrían ser temporales o permanentes. Pero, por encima de todo debemos tener presente que las necesidades educativas tienen su origen en las interacciones alumno-docente-centro educativo y que rara vez nacen exclusivamente del alumno. Al asumir este carácter interactivo las necesidades educativas de los alumnos tienen entonces un carácter relativo.

Por consiguiente, debemos tomar en cuenta el carácter de relatividad de las necesidades educativas, tanto ordinarias como especiales; también deberíamos saber quiénes son los profesores, cuáles son sus características, cuáles son sus necesidades de formación, cuáles han sido sus experiencias. De esta manera, podríamos conocer las características de los actores más importantes del proceso educativo y aumentarían nuestras posibilidades de determinar los puntos de encuentro en las estrategias por ellos desarrolladas, para su intervención pedagógica. Debemos conocer sus preocupaciones, sus puntos de divergencia, los conflictos que se han generado. Esta última parte del trabajo se realizará con el objeto de determinar cuáles serán los apoyos que ellos requieren.

Si tomamos en cuenta el carácter interactivo de las necesidades educativas, en relación con el centro y por ende su carácter de relatividad, deberíamos establecer mecanismos 
más eficientes en la comunidad universitaria, que nos lleven a la reflexión y al análisis de la urgencia de contar con un sistema de apoyo institucional que responda en forma eficiente, que oriente y dé pautas para ofrecer una educación más justa, acorde con las necesidades educativas especiales. De no ser así, el rendimiento académico de los estudiantes con necesidades educativas especiales, particularmente de aquellos(as) cuyas necesidades estén asociadas a una discapacidad, se verán afectados(as) y en algunos casos, podríamos afirmar que alumnos y alumnas con estas características, tendrán que desistir o posponer sus deseos y renunciar a sus derechos de formarse.

Por las razones señaladas, debemos considerar la posibilidad de incluir en forma natural las modificaciones, en la planificación y en la concreción de ese currículo (adecuaciones curriculares), con el fin de atender las necesidades de los estudiantes universitarios. Esto sería estrictamente necesario, si realmente queremos futuros profesionales que entiendan de diversidad, pero que por encima de todo, puedan en un futuro no muy lejano ofrecer servicios profesionales, públicos y privados, a una sociedad naturalmente diversa.

Consideramos que es en este nivel educativo, en el que los estudiantes podrían desarrollar una mayor conciencia de sus derechos como estudiantes a tener la mejor educación posible y la obligación de la Universidad de ofrecer realmente una educación inclusiva y no segregante, con canales de comunicación interactivos y más efectivos, colaborar de esa forma a ofrecer una educación de mejor calidad para todos. 


\section{¿Qué debemos saber? \\ Bases teóricas conceptuales}

\section{Evolución histórica desde la segregación hasta la inte- gración}

A lo largo del tiempo, variadas concepciones sobre desarrollo han sido estudiadas y dentro de ellas se destacan fundamentalmente el preformismo, el predeterminismo, el evolucionismo y el interaccionismo, los cuales se pueden relacionar claramente con los conceptos de deficiencia y discapacidad.

Podemos entonces hacer una relación histórica de los conceptos de desarrollo y deficiencia, con el surgimiento de la Integración Escolar y su piedra angular el Principio de Normalización. Los estudios del ser humano y los conceptos de deficiencia y discapacidad, se han asociado al aprendizaje con otros conceptos como: evolución, maduración, jerarquización, es decir, con desarrollo. Pero el aprendizaje no sólo refleja la adquisición de comportamientos que comprenden únicamente el desarrollo, sino también, las influencias del medio. Con el interaccionismo, aparece un nuevo paradigma de desarrollo, en esta nueva concepción el desarrollo se toma como el resultado de interacciones muy complejas entre la herencia y el ambiente. Ambos constituyen una unidad dialéctica y evolutiva. Feuerstein (1980), afirma que el rendimiento escolar bajo, es debido al uso ineficaz de las funciones mentales que son al mismo tiempo requisitos previos para un funcionamiento cognitivo adecuado. Este uso ineficaz de las funciones mentales que algunas veces no han sido desarrolladas por la ausencia o carencia de experiencias de aprendizaje mediado de buena calidad. 
Bajo esta visión interaccionista, se ve la minusvalía ya no como una condición o característica personal e inherente al individuo, sino como una función de la relación entre la persona con una deficiencia, quien presenta una discapacidad y el ambiente con sus barreras, sean éstas: culturales, físicas, o sociales, que le impiden una total participación, convirtiéndolo o convirtiéndola en una persona minusválida.

$\mathrm{Al}$ asumir este concepto de deficiencia, el potencial hereditario es susceptible de ser modificado y activado por el medio, la herencia negativa o con desviaciones puede ser significativamente influida a través de intervenciones adecuadas del medio. Surge así el Principio de Normalización.

En las postrimerías del siglo XX un halo de esperanza se abre con nuevas tendencias epistemológicas, nuevos enfoques y perspectivas teóricometodológicas y de movimientos sociales en pro de la diferencia, la heterogeneidad, la singularidad, la cooperación, el respeto, la participación. La inclusión y no la exclusión empieza a manifestar sus exigencias en lo educativo como expresión de un nuevo proyecto social, político e ideológico que se perfila como condición para la vida. Debemos ser conscientes de que para asumir el reto que representa el trabajo con jóvenes adultos que manifiestan algunas características particulares, sean estas físicas, sensoriales, emocionales, sociales, que inciden en sus formas de accesar al aprendizaje y al conocimiento, los profesionales requeriremos de un desarrollo y un proceso formativo, que nos permita generar actitudes positivas hacia ellos y en ellos.

Para asumir seriamente el reto que representa la formación profesional de nuestros futuros ciudadanos, los profesionales universitarios requerimos entender que la diversidad 
supone una lógica inclusiva, que incorpore la singularidad, la particularidad, relaciones y procesos, es decir, supone una nueva perspectiva filosófica, política, ideológica y ética.

En nuestros días, la posibilidad de ofrecer una educación de calidad para todos y todas los(las) universitarios (as) desde la perspectiva de la diversidad, nos haría cuestionarnos constantemente, casi diariamente, el para qué estamos educando, cuál es la razón fundamental de la educación, qué significa realmente educar, en un mundo tan cambiante como en el que vivimos hoy día.

\section{Principio filosófico de normalización}

Wolfensberger (1986) define el Principio de Normalización como: "la utilización de medios que sean tan culturalmente normativos como sea posible, para establecer, propiciar o apoyar conductas, apariencias e interpretaciones que sean tan culturalmente normativas como sea posible".

La normalización significa establecer relaciones íntimas, positivas e interpersonales entre ciudadanos comunes y aquellos que tienen una deficiencia o que han sido devaluados por otros motivos. No basta entonces con compartir el mismo espacio físico, es necesario normalizar el entorno, reconociendo y aceptando las diferencias.

La normalización lleva implícitas las estrategias de integración. La integración es considerada como un medio y una meta, es de esta manera un correlato directo del Principio de Normalización, al interactuar, se retroalimentan. "Estar integrado, desde una perspectiva psico-social, supone participar con .... interactuar, formar parte de grupos diversos... Ello implica el establecimiento de canales de comunicación 
entre los actores del campo social, previa adopción de valores y códigos propios de parte de los que se integran". (Verdugo, 1990)

El proceso de integración, entonces, es un proceso de dos vías: por un lado preparar a la sociedad para que integre a las personas discapacitadas y por el otro, preparar a las personas discapacitadas para que se adapten al medio.

De esta manera la integración al medio educativo o integración escolar, constituye un componente importante de este proceso. El proceso de integración es, entonces, el resultado de una interacción e interinfluencia entre diversos componentes y se basa en el principio de normalización. Es el ejercicio del derecho a ser diferente al que le da posibilidades a la persona con discapacidad a poner en práctica los demás derechos humanos.

La adecuación del currículo constituye una medida de atención a la diversidad, concretamente, esta es la estrategia más comúnmente establecida para afrontar la integración escolar de los alumnos y las alumnas con necesidades educativas especiales. Es de esperar que esta estrategia de planificación y actuación de los y las docentes universitarias, como cualquier otra, debe estar situada en el contexto institucional y amparada por un proyecto organizativo realizado en función de un amplio conocimiento y análisis de la realidad a la cual está dirigido.

\section{¿Qué debemos saber hacer?}

Es muy importante reflexionar en relación con la posibilidad de desarrollar un proyecto educativo universitario que promueva y atienda la diversidad de su alumnado para 
poder ofrecer una educación no discriminatoria que compense las dificultades de los alumnos y respete las diferencias individuales, que a su vez favorezca la integración de alumnos con necesidades educativas especiales asociadas a discapacidad y que por encima de todo, promueva el uso de estrategias metodológicas que favorezcan el trabajo cooperativo y colaborativo y el respeto a las diferencias. Para ello, será necesario derivar unas líneas maestras que orienten la reflexión en el equipo de docentes universitarios.

\section{¿Qué podemos hacer los y las docentes en la Universidad?}

- Ofrecer apoyo psicopedagógico a los estudiantes con necesidades educativas especiales, que lo soliciten. Entendemos por apoyo psicopedagógico el asesoramiento en la elaboración, toma de decisiones y gestión de los currículos, sus contenidos, organización y evaluación, es decir es un dinamizador de las tareas educativas para ofrecer respuestas a la diversidad (Torres, 1997).

- Identificar las necesidades que se generan y los conflictos que enfrentan los profesores universitarios, derivadas de las interacciones de enseñanza y aprendizaje, cuándo deben atender estudiantes con necesidades educativas especiales.

- Identificar estrategias innovadoras que desarrollan los profesores universitarios para favorecer la integración de sus alumnos con necesidades especiales.

- Formular programas de apoyo psicopedagógico para profesores universitarios interesados en la atención de los estudiantes con necesidades educativas especiales.

- Favorecer la retroalimentación de nuestro quehacer y del plan de estudios de la carrera de Educación Especial a través de la participación de profesores y alumnos. 
- Llevar a cabo valoraciones de los potenciales de los alumnos y de sus contextos.

- Valorar las necesidades de formación de los docentes para atender la diversidad del aula.

- Intentar que las modificaciones al currículo lo aparten lo menos posible de la programación regular, o de los planteamientos comunes.

- Implementar adecuaciones metodológicas con estrategias y técnicas variadas y diversificadas de trabajo cooperativo y colaborativo.

- Utilizar adecuaciones metodológicas en los procesos de evaluación, en donde podemos combinar criterios de realidad y éxito.

- Intentar conocer los conocimientos que él o la alumna tiene acerca de la temática en discusión y determinar qué es lo que deseamos conseguir al final de cada sesión.

- Desarrollar interdependencia profesional con estrategias que permitan la continuidad.

- Conformar equipos de apoyo y redes de apoyo institucional.

\section{¿Para qué lo hariamos?}

Una forma de cambiar nuestras actitudes es conociendo mejor nuestra condición humana, es entendiendo mejor nuestra diversidad. Es entendiendo que lo diverso se debe atender en forma natural, en forma diversificada. Las adecuaciones al currículo no son más que una forma de ser coherentes con nosotros mismos.

Es nuestra intención tratar de establecer la relación que debe existir entre nuestras vivencias como alumnos de una institución, entre nuestro proceso educativo y nuestro desempeño como futuro profesional. 
Quisiéramos creer que si nuestros alumnos universitarios reciben atención a sus necesidades educativas, ordinarias o especiales, de una forma natural, tomando en consideración únicamente su característica de ser humano diverso, único, irrepetible, con capacidades y necesidades únicas, será posible que él o ella, profesionales en un futuro cercano, conozcan realmente la diversidad humana y la entiendan, para aceptarla y poder convivir con ella en armonía, en un mundo mejor para todos.

\section{Consideraciones finales}

Quisiéramos en estas consideraciones finales valernos de algunos comentarios externados por académicos universitarios en un foro de discusión vía correo electrónico, convocado por la Máster Catarina Goldoni en septiembre de 1999, como parte de un trabajo de graduación bajo el título Educación en y desde la diversidad, atención a las necesidades educativas especiales de los y las estudiantes con discapacidad en la Universidad Nacional. Nos parece que estos comentarios recogen el sentir de académicos universitarios conscientes de la necesidad de un cambio de nuestra forma de percibir las diferencias humanas y por sobre todo de nuestras formas de atenderlas desde la docencia universitaria; para ello es necesario continuar con el análisis y la reflexión de estos temas, que nos toca a todos y a todas las universitarias, pues todos y todas de una u otra forma somos parte de ese mundo de diversidad.

- Algunas decisiones en relación con los diagnósticos clínicos y educativos no son más que un descalabrado fracaso que pone de manifiesto que la ceguera no está en los ojos sino en los marcos simbólicos y éticos a partir de los cuales se aprecia y se juzga el comportamiento de los demás. (Danilo Pérez Zumbado, 1999). 
- No hay duda que deberíamos cuestionar nuestras prácticas y modalidades de enseñanza que nos permita cada vez más lograr una educación de calidad que atienda la diversidad y respete la diferencia.

- Cuando atendemos la diversidad nos enriquecemos con la diferencia (Alvarado, 1999), brillamos en las artes, los deportes, las ciencias.

- En la medida que aprendamos a ser más incluyentes, es decir en que se encuentren respuestas específicas a las peculiaridades de cada quien, más cerca estaremos del grado de diversidad en el que la universidad puede ser fructífera e indispensable. Soñamos así con una universidad diversa.

- La atención a la diversidad de nuestras aulas supone de una nueva concepción de educación, una nueva concepción de universidad y por supuesto de ajustes y modificaciones a las estrategias de enseñanza y de aprendizaje.

- Debemos crear foros de discusión para discutir los fundamentos teóricos y metodológicos de las adecuaciones al currículo, para el análisis y la conceptualización de términos fundamentales como: educación, currículo, diversidad, necesidad educativa especial, otros.

- La universidad como otra institución educativa no tiene claro hacia dónde orientar sus acciones (Tedezco citado por Goldoni, 1999).

- Un rasgo de la nueva Universidad debería ser no sólo la tolerancia, ni sólo la solidaridad, sino también la liberación de la potencia escondida que encierra lo singular y lo diferente. (Alvarado, 1999).

- Preparar para la vida y no para el empleo, este es el reto al que nos enfrenta una educación que atiende la diversidad del signo que sea y particularmente, la asociada a personas con discapacidad. (Goldoni, 1999). 
- Es responsabilidad nuestra, de los que tenemos discapacidades menos notorias, hacer que los ciegos, sordos, parapléjicos reciban una educación que exija de ellos el máximo desarrollo de sus potencialidades y la mejor calidad. Para lograr esto nosotros muchas veces tenemos que hacer un mayor esfuerzo y sobretodo adecuar nuestras técnicas de enseñanza. (Coen, E., 1999).

Como podemos ver, en estos comentarios encontramos ideas fundamentales ofrecidas por docentes universitarios que nos invitan a continuar el análisis y la reflexión en relación con las adecuaciones del currículo, como una forma de entender y atender la diversidad presente en nuestras aulas universitarias. 


\section{BIBLIOGRAFÍA}

Sánchez, A. y Torres, J. 1996. Educación Especial: Una perspectiva curricular, organizativa y profesional. Pirámide. Madrid.

Feurstein, R. 1987. El pensador Remedial. Revista Aula № 56 Grao Educación, Barcelona.

Lou, M. y López, N. 1998. Bases psicopedagógicas de la Educación Especial. Pirámide. Madrid.

Verdugo, M. 1995. Aula: Innovación Calidad de vida y educación especial. Presente y futuro de la educación especial. Número 45. Grao Educación. Barcelona.

Correo electrónico Universidad Nacional, noviembre 1999. 